\title{
The new AJCC/TNM Staging System (VIII ed.) in papillary thyroid cancer: clinical and molecular impact on overall and recurrence free survival
}

\author{
Giulia Sapuppo ${ }^{1}$, Martina Tavarelli ${ }^{2}$, Gabriella Pellegriti ${ }^{2}$ \\ ${ }^{1}$ Endocrinology, Department of Clinical and Experimental Medicine, University of Catania, Garibaldi-Nesima Medical Center, Catania, Italy; \\ ${ }^{2}$ Endocrinology, Garibaldi-Nesima Medical Center, Catania, Italy \\ Correspondence to: Gabriella Pellegriti, MD, PhD. Endocrinology, Garibaldi-Nesima Medical Center, Via Palermo 636, 95122 Catania, Italy. \\ Email: g.pellegriti@unict.it; gabriellapellegriti@hotmail.com. \\ Provenance and Peer Review: This article was commissioned by the editorial office, Annals of Translational Medicine. The article did not undergo \\ external peer review. \\ Comment on: Kim K, Kim JH, Park IS, et al. The Updated AJCC/TNM Staging System for Papillary Thyroid Cancer (8th Edition): From the \\ Perspective of Genomic Analysis. World J Surg 2018;42:3624-31.
}

Submitted Jan 20, 2020. Accepted for publication Feb 20, 2020.

doi: $10.21037 / \mathrm{atm} .2020 .03 .80$

View this article at: http://dx.doi.org/10.21037/atm.2020.03.80

Differentiated thyroid cancer (DTC) is the most common endocrine malignancy $(1,2)$ and over $85 \%$ of DTC cases have a papillary histotype (PTC). Its incidence, relatively stable until the early 1990s, has rapidly grown in recent decades, more than any other cancer (3), due mostly to an increase of low-risk thyroid cancer (TC).

DTC is generally associated with an excellent prognosis: the 5 -year survival rate is near $100 \%$ for localized disease, $98 \%$ for regional disease and $56 \%$ for metastatic disease.

Additionally, the death rate for TC has increased slightly in recent years, from 0.50 (per 100,000) in 2007 to 0.54 in 2016, in spite of earlier diagnosis and better treatment (https://www.cancer.org/content/dam/cancer-org/ research/cancer-facts-and-statistics/annual-cancer-factsand-figures/2019/cancer-facts-and-figures-2019.pdf) (4). Although DTC has a good prognosis, in some patients tumor behavior is aggressive and associated with poor outcome. A major issue, therefore, is finding characteristics and criteria that identify these tumors for appropriate management.

Recently American guidelines (5) introduced a new risk stratification system with additional prognostic variables for tailored management. Moreover, the TNM (Tumor, Node, Metastasis) classification was changed in 2018 to better predict DTC survival. Most of the changes in the 8th edition (TNM-8) downstaged a significant number of patients into lower stages to more accurately reflect their low risk of dying.

The changed American Thyroid Association (ATA) risk stratification and TNM staging have a significant impact on both the initial therapeutic decision and subsequent followup management. For this purpose, as recently suggested (6), molecular analysis data would be helpful in identifying the most aggressive DTC cases, thus influencing treatment decision making and subsequent follow-up.

Recently, Lee and colleagues describe a retrospective cohort of 505 PTC cases analyzed from The Cancer Genome Atlas (TCGA) database portal (7).

The objectives of the study were:

(I) To assess the accuracy of TNM-8 compared to TNM-7 in predicting PTC overall survival (OS) and recurrent free survival (RFS);

(II) To compare gene expression data, copy number alterations and somatic mutation profiles according to age at time of cancer diagnosis in order to evaluate the efficacy of TNM-8, not only on a clinical but also a genomic level.

The authors analyzed four major points:

Age cut-off and risk factors: PTC patients were subdivided on the basis of age at diagnosis using the previous cut-off of 45 years (TNM-7) and the new one of 55 years (TNM-8). Among the analyzed risk factors, 
multifocality, site of tumor and BRAF gene mutation showed no significant difference in outcome for either age group whereas a statistical difference was found for minimal extrathyroidal extension (mETE) using both age cut-offs. Male gender and larger tumors were predictors of the worst outcome using the 55-year cut-off.

Many reports analyzed the effect of changing the age cut-off, showing that an age cut-off higher than 45 years was a better indicator of cancer-related death risk.

Tam et al. (8) evaluated the effect on disease-specific survival (DSS) and OS of downstaging due to different age cut-offs in a retrospective series of 2,579 DTC. The 10 -year DSS of the 45-54-year age group was $97.6 \%$, which is higher (but not statistically significant) than for patients aged $<45$ years and lower than for patients aged $\geq 55$ years (significant only with univariate analysis). They concluded that the survival discrimination power between TNM-7 and TNM-8 systems was no different and that both editions have the same ability to estimate survival among the stages.

In contrast, results from Kim et al. (9), analysing a large cohort of 3,176 DTC patients, and Kim et al. (10), including 1,613 DTC patients, suggested that TNM-8 has a higher ability to differentiate patients of different stages and therefore to predict DSS.

Regarding the choice of the new age cut-off, Mazurat and colleagues (11) showed an optimal age cut-off of 55 years but Kim et al. (12), with 35,323 patients from the Surveillance, Epidemiology and End Results (SEER) database, found that the optimum cut-off point for diseaserelated death was 57 years.

Moreover, as shown by several studies, mortality increases progressively with advancing age and any single cut-off point for age is less 'performant' than models that consider age as a continuous variable.

mETE is a controversial prognostic factor and several studies have evaluated its role on DSS and OS. Some authors (13-16) revealed similar clinical outcomes of patients with mETE and those with no ETE. However, Castagna et al. (17) showed poorer outcome (persistent structural or recurrent disease or tumor-related death) in patients with ETE compared with tumors larger than $1.5 \mathrm{~cm}$ with negative margins ( $11.8 \%$ vs. $5.1 \%)$, concluding that only small mETE tumors should be classified as lowrisk tumors.

Tran et al. (18) found no association between tumor size and RFS in patients aged $<55$ years but that it was an independent predictor in patients aged $\geq 55$ years, concluding that the impact of tumor size on RFS was limited to older patients.

We agree with the better prognostic accuracy of the shifted age cut-off supported by several studies (10-12).

However, with regard to the mETE prognostic value we are more cautious as it still remains controversial. Recently an expansion of TNM-8 has been published (telescoping) with the objective of collecting additional data without altering the definitions of the current TNM categories in order to better classify each tumor category according to the presence or absence of $\mathrm{mETE}$ and to test the subcategories for prognosis and treatment planning considerations. In the next few years, we shall have more information on the importance of mETE for each tumor category from several groups of authors.

\section{RFS and OS in both age groups}

RFS was not statistically different using the cut-off of 45 years but it was significantly worse for older patients with the cut-off at 55 years. OS was statistically lower for older patients using both age cut-offs.

Kim et al. (10) showed that age $\geq 55$ years (but not 45 54 years) was a significant predictor of recurrence and overall mortality $(\mathrm{P}<0.005)$. Both TNM-7 and TNM8 were predictive of RFS and OS $(\mathrm{P}<0.001)$ but TNM8 better discriminated the tumor classification and overall TNM stage than TNM-7.

Nixon (19) showed that the shift of the age cut-off to 55 years also improved prediction of the 10-year DSS; survival improved from $99 \%$ to $76 \%$ for stages I-IV at an age cutoff of $\geq 45$ years and from $99 \%$ to $67 \%$ at an age cut-off of $\geq 55$ years, respectively.

\section{Comparison of TNM-7 and TNM-8}

Among 493 patients, $41 \%$ were downstaged into lower stages and unavoidably more cases of recurrences and deaths were found in the lower stages. In particular, $17 \%$ of patients downstaged from stage III to stage II had recurrent disease; $25 \%$ of cases downstaged from stage IV to III, $13.6 \%$ from stage IV to II and $18.4 \%$ from stage IV to stages III and II died for PTC.

The Kaplan-Meier plot for stage-dependent RFS and OS showed a statistically significant value for both editions. However, RFS had a more significant $\mathrm{P}$ value using the TNM-8 staging system than the TNM-7 system and for OS the value was higher for TNM-7 (but significant value for both eds.). 
Recently, several studies have been conducted to compare TNM-7 and TNM-8, and better predictability in patients with DTC by TNM-8 has been suggested $(10,20,21)$. When TNM-8 is applied, a significant number of patients with DTC are reclassified to lower stages and more accurate survival predictions are provided compared with TNM-7. Therefore, the changes in TNM- 8 are expected to provide a more realistic assessment of disease mortality in high-risk patients.

TNM-8, on the one hand, suggests an improved allocation of patients at high risk of dying from DTC into more advanced TNM stages and, on the other hand, induces a wrong belief of less aggressive disease. However, it should be emphasized that the risk of death is not always related to the risk of recurrence in many patients.

Tam et al. (8) showed that the 10-year DSS for stages IIV for TNM-7 ranged from $100 \%$ to $82.6 \%(\mathrm{P}<0.001)$ and for TNM-8 from $99.8 \%$ to $71.9 \%(\mathrm{P}<0.001)$. The 10 -year OS for stages I-IV based on TNM-7 ranged from $95.8 \%$ to $59.7 \%$ and for TNM-8 from $94.3 \%$ to $34.6 \%$. The power of survival prediction for DSS in TNM-7 and TNM-8 is similar, although the 10-year DSS appears more appropriate between stages using the updated TNM-8.

Kim et al. (10), analysing 1,613 patients, showed that using TNM-8, $38 \%$ of patients were reclassified into lower stages and $63 \%$ of patients with T3 classification were restaged as $\mathrm{T} 1$ or $\mathrm{T} 2$. The DSS results for patients in stages III and IV according to TNM-8 were worse than those according to TNM-7 (98.8\% and $83.2 \%$, respectively, for TNM-7; 72.3\% and 48.6\%, respectively, for TNM-8). They concluded that TNM- 8 improves the prediction of both recurrence and survival in patients with PTC from the previous TNM-7 staging system.

In the study by Shteinshnaider et al. (21), the proportion of intermediate/high-risk patients in stages I-II according to TNM-8 increased considerably compared to TNM7. Patients reclassified according to TNM-8 in stage II had more lymph node metastases, more recurrence risk, more reoperations, more persistency of disease and a non-significant increase in disease-specific mortality compared to TNM-7. This study underlines that TNM-8 provides a more accurate system to discriminate mortality and persistence in DTC patients but that the severity of disease, especially in the 45-55-year age group and in stage II patients, should not be underestimated following the important down-staging of these patients.

From our point of view, although the new TNM-8 in comparison to TNM-7 would seem to better discriminate mortality, the significant downstaging could underestimate the severity of disease in many patients and cause a nonnegligible treatment burden, as for patients with laterocervical metastases at diagnosis (22), especially when of large size and numerous (23).

\section{Specific gene signature among the three groups of patients $(<45,45-55$ and $\geq 55$ years)}

Lee and colleagues found that there were no specific molecular subtypes between the three age groups. There were 14 specific genes found in patients aged $<45$ years, none in patients aged $45-54$ years and 103 in patients aged $\geq 55$ years. These data showed that raising the cut-off age from 45 to 55 years more effectively predicts the disease prognosis of PTC and supports the use of TNM-8, making it clinically and genetically appropriate. No difference was found in copy number alteration or somatic mutation patterns. Moreover, there was no statistically significant difference in seven of the most frequently mutated genes (BRAF, HRAS, NRAS, etc.) according to each age group.

In summary, the authors evaluated the potential signalling pathways activated in each age group of patients: for example, those older than 55 years had alterations in the sirtuin signaling pathway, ATM signaling, the FXR/RXR activation pathway and the transforming growth factor- $\beta$ pathway.

This study is the first to evaluate clinical and gene expression data in all patients and according to previous and recent age cut-offs and shows clinical and genetic evidence supporting the age of 55 years as being the better cut-off.

There are several limitations to this excellent study. It is a retrospective analysis using TCGA data and the impact of these specific genes in different ages cannot be defined due to the lack of correlation between molecular and clinical data.

Currently, none of the mortality risk systems incorporate molecular testing results. This may need to be re-evaluated because several studies have shown that molecular testing, including BRAF V600E, TERT and TP53 or combinations of markers, has an important impact on the risk of recurrence and mortality.

Xing et al. (24) showed in 1,849 PTC patients that the presence of a $B R A F$ mutation was associated with increased disease-specific mortality, although this was not significantly associated with mortality in a multivariate analysis. However, a significant interaction between $B R A F$ mutation and several conventional clinicopathological risk 
factors was seen: lymph node metastases, distant metastases and American Joint Committee on Cancer (AJCC) stage IV disease.

In a systematic review and meta-analysis of 14 publications (25), including 2,470 PTC patients, the $B R A F$ mutation was associated with a significantly higher risk of recurrence than $B R A F$ wild-type tumors.

Two other molecular markers, TP53 and TERT mutations, appear to confer an increased risk of tumor recurrence and tumor-related mortality. In one study that analyzed more than 400 DTC cases (26), the presence of a TERT mutation was found to be an independent predictor of mortality.

Another study (6) showed that the PTC recurrence rate for patients with coexisting $B R A F$ and TERT mutations was significantly higher than that associated with either mutation alone, demonstrating an incremental and synergistic effect of the coexisting two mutations.

Recently Gan and colleagues (27) investigated the significance of the $B R A F \mathrm{~V} 600 \mathrm{E}$ mutation in predicting prognostic and aggressive clinicopathological characteristics according to a new age-based stratification. In the $\geq 55$-year age group, $B R A F \mathrm{~V} 600 \mathrm{E}$ was found to be significantly associated with aggressive PTC characteristics, including tumor size, PTC subtype, radioactive iodine dose, follow-up time, recurrence, recurrence risk stage, advanced tumor stage, advanced node stage and AJCC stage III/IV (all $\mathrm{P}<0.05)$. Recurrence-free survival rate was statistically different in the $\geq 55$-year age group $(\mathrm{P}=0.04)$ but there was no significant difference in the $<55$-year age group $(\mathrm{P}=0.76)$, according to the $B R A F \mathrm{~V} 600 \mathrm{E}$ mutation status. They therefore concluded that the $B R A F$ V600E mutation was found to better predict aggressive and recurrent PTC based on age stratification with the cut-off age of 55 years.

In a recent paper, Yan and colleagues (28) analyzed the relationship between $B R A F$ V600E and clinical features in PTC; with regard to age categories, they showed a significant difference of $B R A F \mathrm{~V} 600 \mathrm{E}$ incidence between patients aged $\leq 45$ and $>45$ years $(79.7 \%$ vs. $88.4 \%$, $\mathrm{P}<0.001$ ), concluding that PTC patients were more prone to be $B R A F \mathrm{~V} 600 \mathrm{E}$ positive with increasing age but that $B R A F \mathrm{~V} 600 \mathrm{E}$ has no independent prognostic value of risk in connection with outcome.

Regarding the impact of ATM (critical in the process of recognizing and repairing DNA lesions) and the FXR/RXR pathways (members of the nuclear family of receptors and key players in the control of numerous metabolic patbways), many reports have supported the association with PTC prognosis and survival (29-31).

Giaginis and colleagues (31) showed that enhanced farnesoid X receptor (FXR) was more frequently observed in PTC compared with hyperplastic nodules, and that in malignant lesions high levels were associated with capsular and vascular invasion, increased follicular cell proliferative rate, larger tumor size, presence of lymph node metastases, lymphatic invasion and increased recurrence rate risk.

Because of the clinical implications of this incremental improvement in risk stratification of $B R A F$ and other mutations such as TERT, research on mutational status is not routinely recommended but could help to refine risk estimates when interpreted in the context of other clinicopathological risk factors.

Very recently, the importance of the impact of molecular signatures has been increasing and will allow a more specific detection of well-differentiated TC cases that have high risks of tumor recurrence and cancer-related mortality.

To date, none of the current molecular markers were considered to have sufficient independent prognostic significance to be included in the new staging system; moreover, Lee and colleagues do not indicate the mean follow-up of analyzed patients, which is a crucial point to better establish the real impact of mutation status on shortand long-term follow-up. It may be the case, mainly for some tumors with specific mutations, that a long follow-up is needed to disclose their aggressiveness.

In conclusion, TNM-8 staging should have greater accuracy in identifying patients at higher risk of dying of TC, but careful follow-up is also needed for downstaged patients. Even though molecular profiling of tumors has the potential to better estimate cancer aggressiveness and other risk factors, further studies are needed.

\section{Acknowledgments}

Funding: None.

\section{Footnote}

Conflicts of Interest: All authors have completed the ICMJE uniform disclosure form (available at http://dx.doi. org/10.21037/atm.2020.03.80). The authors have no conflicts of interest to declare.

Ethical Statement: The authors are accountable for all 
aspects of the work in ensuring that questions related to the accuracy or integrity of any part of the work are appropriately investigated and resolved.

Open Access Statement: This is an Open Access article distributed in accordance with the Creative Commons Attribution-NonCommercial-NoDerivs 4.0 International License (CC BY-NC-ND 4.0), which permits the noncommercial replication and distribution of the article with the strict proviso that no changes or edits are made and the original work is properly cited (including links to both the formal publication through the relevant DOI and the license). See: https://creativecommons.org/licenses/by-nc-nd/4.0/.

\section{References}

1. Davies L, Welch HG. Current thyroid cancer trends in the United States. JAMA Otolaryngol Head Neck Surg 2014;140:317-22.

2. Shin HR, Masuyer E, Ferlay J, et al. Cancer in Asia Incidence rates based on data in cancer incidence in five continents IX (1998-2002). Asian Pac J Cancer Prev 2010;11 Suppl 2:11-6.

3. Pellegriti G, Frasca F, Regalbuto C, et al. Worldwide increasing incidence of thyroid cancer: update on epidemiology and risk factors. J Cancer Epidemiol 2013;2013:965212.

4. Facts \& Figures annual report. American Cancer Society. Available online: (https://www.cancer.org/content/ dam/cancer-org/research/cancer-facts-and-statistics/ annual-cancer-facts-and-figures/2019/cancer-facts-andfigures-2019.pdf

5. Haugen BR. 2015 American Thyroid Association Management Guidelines for Adult Patients with Thyroid Nodules and Differentiated Thyroid Cancer: What is new and what has changed? Cancer 2017;123:372-81.

6. Xing M, Liu R, Liu X, et al. BRAF V600E and TERT promoter mutations cooperatively identify the most aggressive papillary thyroid cancer with highest recurrence. J Clin Oncol 2014;32:2718-26.

7. Kim K, Kim JH, Park IS, et al. The Updated AJCC/ TNM Staging System for Papillary Thyroid Cancer (8th Edition): From the Perspective of Genomic Analysis. World J Surg 2018;42:3624-31.

8. Tam S, Boonsripitayanon M, Amit $M$, et al. Survival in Differentiated Thyroid Cancer: Comparing the AJCC Cancer Staging Seventh and Eighth Editions. Thyroid 2018;28:1301-10.
9. Kim TH, Kim YN, Kim HI, et al. Prognostic value of the eighth edition AJCC TNM classification for differentiated thyroid carcinoma. Oral Oncol 2017;71:81-6.

10. Kim M, Kim WG, Oh HS, et al. Comparison of the Seventh and Eighth Editions of the American Joint Committee on Cancer/Union for International Cancer Control Tumor-Node-Metastasis Staging System for Differentiated Thyroid Cancer. Thyroid 2017;27:1149-55.

11. Mazurat A, Torroni A, Hendrickson-Rebizant J, et al. The age factor in survival of a population cohort of well-differentiated thyroid cancer. Endocr Connect 2013;2:154-60.

12. Kim SJ, Myong JP, Suh H, et al. Optimal Cutoff Age for Predicting Mortality Associated with Differentiated Thyroid Cancer. PLoS One 2015;10:e0130848.

13. Jin BJ, Kim MK, Ji YB, et al. Characteristics and significance of minimal and maximal extrathyroidal extension in papillary thyroid carcinoma. Oral Oncol 2015;51:759-63.

14. Moon HJ, Kim EK, Chung WY, et al. Minimal extrathyroidal extension in patients with papillary thyroid microcarcinoma: is it a real prognostic factor? Ann Surg Oncol 2011;18:1916-23.

15. Woo CG, Sung CO, Choi YM, et al. Clinicopathological Significance of Minimal Extrathyroid Extension in Solitary Papillary Thyroid Carcinomas. Ann Surg Oncol 2015;22 Suppl 3:S728-33.

16. Ito $\mathrm{Y}$, Tomoda $\mathrm{C}$, Uruno $\mathrm{T}$, et al. Minimal extrathyroid extension does not affect the relapse-free survival of patients with papillary thyroid carcinoma measuring $4 \mathrm{~cm}$ or less over the age of 45 years. Surg Today 2006;36:12-8.

17. Castagna MG, Forleo R, Maino F, et al. Small papillary thyroid carcinoma with minimal extrathyroidal extension should be managed as ATA low-risk tumor. J Endocrinol Invest 2018;41:1029-35.

18. Tran B, Roshan D, Abraham E, et al. The Prognostic Impact of Tumor Size in Papillary Thyroid Carcinoma is Modified by Age. Thyroid 2018;28:991-6.

19. Nixon IJ, Wang LY, Migliacci JC, et al. An International Multi-Institutional Validation of Age 55 Years as a Cutoff for Risk Stratification in the AJCC/UICC Staging System for Well-Differentiated Thyroid Cancer. Thyroid 2016;26:373-80.

20. Pontius LN, Oyekunle TO, Thomas SM, et al. Projecting Survival in Papillary Thyroid Cancer: A Comparison of the Seventh and Eighth Editions of the American Joint Commission on Cancer/Union for International Cancer Control Staging Systems in Two Contemporary National 
Patient Cohorts. Thyroid 2017;27:1408-16.

21. Shteinshnaider M, Muallem Kalmovich L, Koren S, et al. Reassessment of Differentiated Thyroid Cancer Patients Using the Eighth TNM/AJCC Classification System: A Comparative Study. Thyroid 2018;28:201-9.

22. Sapuppo G, Tavarelli M, Russo M, et al. Lymph node location is a risk factor for papillary thyroid cancer-related death. J Endocrinol Invest 2018;41:1349-53.

23. Randolph GW, Duh QY, Heller KS, et al. The prognostic significance of nodal metastases from papillary thyroid carcinoma can be stratified based on the size and number of metastatic lymph nodes, as well as the presence of extranodal extension. Thyroid 2012;22:1144-52.

24. Xing M, Alzahrani AS, Carson KA, et al. Association between BRAF V600E mutation and mortality in patients with papillary thyroid cancer. JAMA 2013;309:1493-501.

25. Tufano RP, Teixeira GV, Bishop J, et al. BRAF mutation in papillary thyroid cancer and its value in tailoring initial treatment: a systematic review and meta-analysis. Medicine (Baltimore) 2012;91:274-86.

26. Melo M, da Rocha AG, Vinagre J, et al. TERT promoter

Cite this article as: Sapuppo G, Tavarelli M, Pellegriti G. The new AJCC/TNM Staging System (VIII ed.) in papillary thyroid cancer: clinical and molecular impact on overall and recurrence free survival. Ann Transl Med 2020;8(13):838. doi: 10.21037/ atm.2020.03.80 mutations are a major indicator of poor outcome in differentiated thyroid carcinomas. J Clin Endocrinol Metab 2014;99:E754-65.

27. Gan X, Shen F, Deng X, et al. Prognostic implications of the BRAF-V600(E) mutation in papillary thyroid carcinoma based on a new cut-off age stratification. Oncol Lett 2020;19:631-40.

28. Yan C, Huang ML, Li X, et al. Relationship between BRAFV600E and clinical features in papillary thyroid carcinoma. Endocr Connect 2019;8:988-96.

29. Gu Y, Liu X, Yu Y, et al. Association of ATM Gene Polymorphism with PTC Metastasis in Female Patients. Int J Endocrinol 2014;2014:370825.

30. Kang J, Deng XZ, Fan YB, et al. Relationships of FOXE1 and ATM genetic polymorphisms with papillary thyroid carcinoma risk: a meta-analysis. Tumour Biol 2014;35:7085-96.

31. Giaginis C, Tsoukalas N, Alexandrou P, et al. Clinical significance of farnesoid $\mathrm{X}$ receptor expression in thyroid neoplasia. Future Oncol 2017;13:1785-92. 